Comparison between Neutron Counting Experimental Measurements and Simulations: Cosmic Ray Contribution

J. M. Verbeke, N. J. Snyderman, L. F. Nakae

February 21, 2008 
This document was prepared as an account of work sponsored by an agency of the United States government. Neither the United States government nor Lawrence Livermore National Security, LLC, nor any of their employees makes any warranty, expressed or implied, or assumes any legal liability or responsibility for the accuracy, completeness, or usefulness of any information, apparatus, product, or process disclosed, or represents that its use would not infringe privately owned rights. Reference herein to any specific commercial product, process, or service by trade name, trademark, manufacturer, or otherwise does not necessarily constitute or imply its endorsement, recommendation, or favoring by the United States government or Lawrence Livermore National Security, LLC. The views and opinions of authors expressed herein do not necessarily state or reflect those of the United States government or Lawrence Livermore National Security, LLC, and shall not be used for advertising or product endorsement purposes.

This work performed under the auspices of the U.S. Department of Energy by Lawrence Livermore National Laboratory under Contract DE-AC52-07NA27344. 


\title{
Comparison between Neutron Counting Experimental Measurements and Simulations: Cosmic Ray Contribution
}

\author{
J.M. Verbeke, N.J. Snyderman, and L.F. Nakae \\ Lawrence Livermore National Laboratory \\ P.O. Box 808, Livermore, CA 94551
}

\section{Executive Summary}

In this report we model old experimental neutron count distribution data with simulations. Included in the simulations are spontaneous fission induced fission chains and cosmic ray induced neutron signals. The experimental data on highly enriched uranium (HEU) was taken at LLNL at sea level, and on Pu at LANL at 7300 feet in altitude. Cosmic rays can create neutron bursts in high-Z materials, which are enhanced in multiplying Special Nuclear Materials (SNM). For HEU at sea level, comparison between simulations and experimental data show that the largest burst events

are initiated by cosmic rays, not by spontaneous fission of ${ }^{238} \mathrm{U}$. Attempts to quantitatively model the HEU experimental data show that additional contributions from cosmic ray induced neutron background in a concrete room improve the comparison significantly. Cosmic rays interacting with the room create a neutron background that dominates the count rate, and can also initiate fission in HEU; the concrete walls can also shield the highenergy hadrons (protons, neutrons, and pions) that create the largest neutron bursts. For HEU of low multiplication, cosmic rays make it easier to detect HEU, but harder to analyze. For a high multiplication Pu ball, even at altitude, the spontaneous fission-initiated fission chains totally dominate the signal - in this regime cosmic rays are unimportant.

\section{Data and Simulations}

In order to illustrate our capability to identify SNM in storage containers, we show examples of comparisons between experimental data and Monte Carlo simulations. The form of the data is random time gate neutron count distributions. When compared to a Poisson distribution that characterizes a random source, a fissioning source shows an increased (Feynman) variance. We also show the time gate dependent dependence of these distributions, which contains information about moderation of a fission source. The 
examples span a range of possible sources. In a previous report ${ }^{1}$ three examples were presented: i) ${ }^{252} \mathrm{Cf}$, a spontaneous fission source, ii) a highly multiplying $4.5 \mathrm{~kg}$ ball of weapons grade plutonium (Pu ball) moderated by 3" thick polyethylene, and iii) a bare $22 \mathrm{~kg}$ shell of HEU, a very weak neutron source with low multiplication. From the previous results, the simulations of the neutron signal from the bare HEU shell only qualitatively reproduced the experimental results. The experimental count rate of about 6 counts/second was dominated by the background contribution, about 4 counts/second, which was not included in the simulations. The HEU experiment was performed at LLNL, at sea level. The ${ }^{252} \mathrm{Cf}$ experiment, also done at Livermore, is essentially unaffected by this background because the source is too small to have fission induced by cosmic rays, and because the background correction to the count rate is negligible - the ${ }^{252} \mathrm{Cf}$ count rate was over 13,000 counts/second, and the background about 4 count/second. Even for the Pu ball, the agreement was imperfect. For the high multiplying $\mathrm{Pu}$, the agreement between data and simulation was very good, but the largest neutron bursts were not being reproduced by spontaneous fission initiated fission chains alone. The Pu experiments were done at Los Alamos, at 7300 feet altitude. We therefore consider the cosmic ray contribution. Cosmic rays can create neutron bursts in high-Z materials that resemble fission chains ${ }^{3}$. The cosmic ray induced neutron bursts are enhanced by fission chains. More generally cosmic rays create a neutron background that can contribute to count rate, and can also initiate fission chains.

In this report we include simulations of the cosmic ray induced neutron signal for the HEU shell at sea level, and for the Pu ball at 7300 feet altitude. The cosmic ray source included hadrons and muons. (The cosmic ray source was developed at LLNL under a DNDO project ${ }^{2}$. Showers were simulated beginning from the primary cosmic rays incident on the earth's atmosphere.)

\section{HEU}

A bare $22 \mathrm{~kg}$ shell of HEU of 6" outer diameter was simulated. The ${ }^{3} \mathrm{He}$ detector model was taken from the ${ }^{252} \mathrm{Cf}$ modeling of reference 1. Examples of neutron count distributions are shown below. The standard time gate shown is for a $512 \mu$ s time gate. A burst of neutrons created on a submicrosecond time scale must be thermalized before efficient counting with ${ }^{3} \mathrm{He}$. The diffusion time constant of the detector is about $40 \mu \mathrm{s}$, so the 
neutrons counted have independently diffused for this time scale before being captured by the ${ }^{3} \mathrm{He}$; neutrons counted from a single burst are typically spread in time by $\sim 100 \mu \mathrm{s}$. An important characteristic of fission chains is that the number of neutrons created, following an initiation event, has enormous fluctuations from one chain to the next. For HEU, the fission chain initiation rate is so low that the chains are well isolated in time; for most time gates of $512 \mu$ s there are no counts, and when there is a count, there is a good chance to get multiple counts from the same fission chain. A $512 \mu$ s time gate is long enough that it is likely that almost all the neutrons from a single burst are counted within that time gate. Chains are sometimes split between neighboring time gates, though, because of the random imposition of the time gate on the time series of counts. Fission chains are initiated by spontaneous fission, and by cosmic ray interactions.

We first show the simulated contribution from spontaneous fission induced chains only, compared to the experimental data.

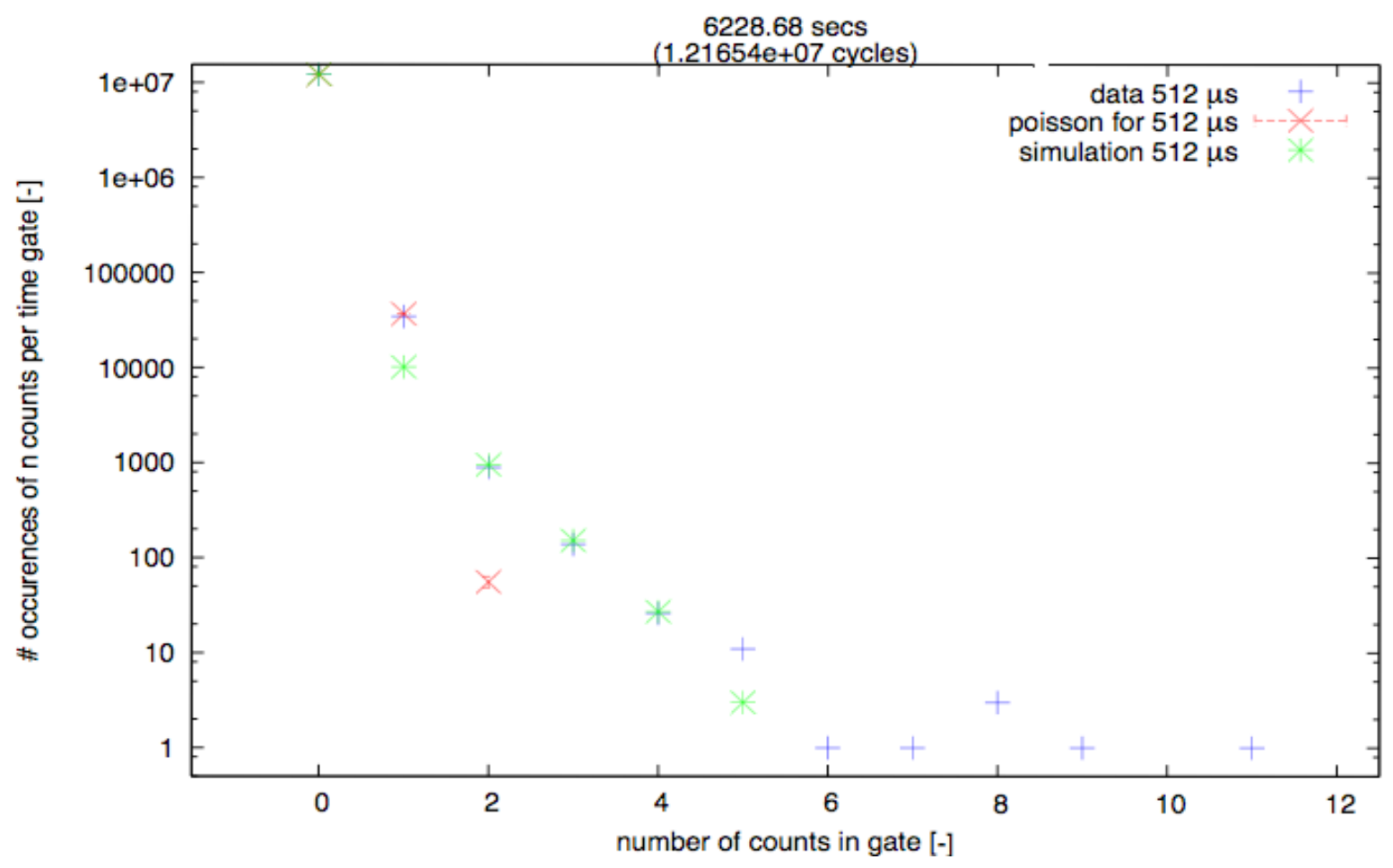

Figure 1: Passive neutron count distribution for a $512 \mu$ s time gate from HEU shell: simulation of spontaneous fission initiated fission chains (green) compared to experimental data (blue). The red points are a Poisson distribution of the same count rate as the experimental data. Both data and simulation show a much broader distribution than Poisson, indicative of larger than random fluctuations. The count time is over 1.7 hours. The experimental data shows occurrences of larger numbers of counts than can not be explained by spontaneous fission initiated chains alone. 
The simulated count distribution from spontaneous fission initiated fission chains cannot account for the complete experimental distribution, although the number of occurrences of $0,2,3,4,5$, and 6 counts is in good agreement. The count rate from the simulation is 2.02 counts/second, while the experimental count rate is 5.9 counts/second. The number of occurrences of 1 count shows the large discrepancy. There is also a large discrepancy in the large number tail of 7 or more counts. The discrepancies are due to background not included. The cosmic rays are an additional source of neutron bursts, due to high-energy hadron interactions on the uranium shell and subsequent enhancement from fission chains. Although the dominant cosmic rays at sea level are muons, they do not significantly contribute to bursts ${ }^{3}$, although as we will discuss below, they do contribute to additional neutron background. A simulation including the cosmic rays, as well as spontaneous fission initiated chains, is shown below.

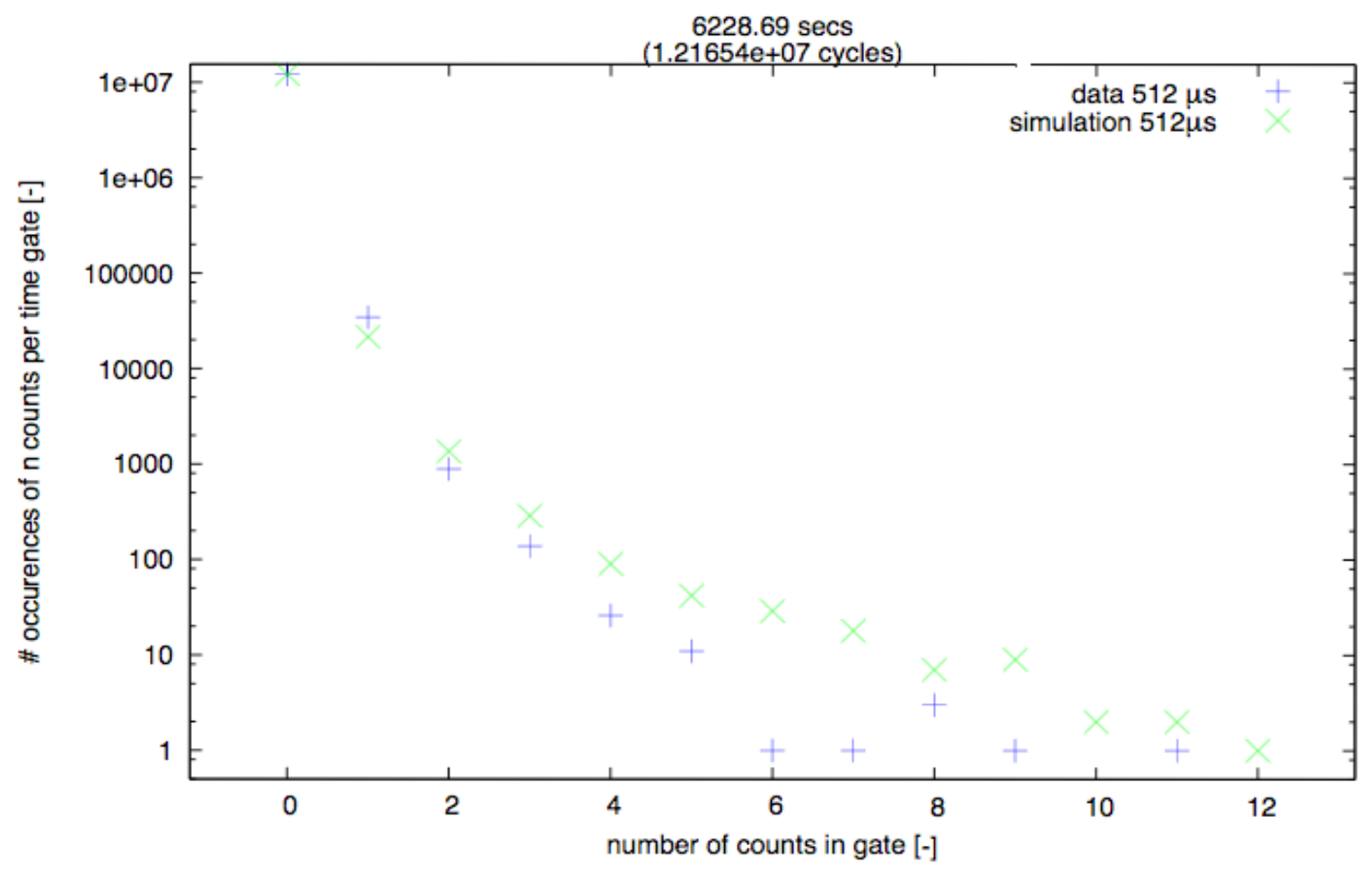

Figure 2: Simulation of cosmic ray induced neutron bursts in bare Tech 1D HEU shell, in addition to spontaneous fission initiated fission chains. The simulated random time gate neutron count distribution (green) for the $512 \mu$ s time gate is again compared to the experimental data (blue). The cosmic ray source at sea level includes protons, neutrons, pions, and muons. The large cosmic ray initiated bursts enhanced by fission chains now give too many occurrences of large bursts. Despite the direct cosmic ray enhancement of larger bursts, the count rate from spontaneous fission and cosmic ray initiated events is now 4.21 counts /second, still less than the measured count rate of 5.9 counts/second. 
Direct cosmic ray interactions with the HEU create a number of occurrences of large bursts that is now larger than the data. The simulated count rate from combined spontaneous fission and cosmic ray initiated events, though, is still less than the experimental data by about 1.7 counts/second. The cosmic rays that have sufficient energy and rate to have created the large bursts typically have energy of about $1 \mathrm{GeV}$. These cosmic rays can be affected by the presence of a room, which we next add to the simulations. Although muons are not significantly affected by the presence of a concrete room, the cosmic ray hadrons can lose enough energy for their burst contribution to be strongly reduced, as shown in the Figure 3.



Figure 3: Simulated neutron random count distribution for bare Tech 1D HEU shell for $512 \mu$ s time gate (green), compared to experimental data (blue). As well as well as spontaneous fission initiated fission chains, the simulation includes cosmic rays at sea level, and a concrete room. Apart from the single occurrence of 20 counts in the simulation in about 1.7 hours count time from a rare highenergy cosmic ray, the room decreases the contribution of the high-energy cosmic rays to the high multiplicity tail, and increases the overall count rate to 5.65 counts/second. 
The room in which the experiment was performed had thick concrete walls and floor. It was only modeled in the most qualitative way. Nevertheless, the simulations show that a room can have a significant affect on passive HEU neutron counting data. A room is a leaky neutron box in which cosmic rays, including the dominant muons, are creating a neutron background. These background neutrons shine directly on the detectors, and can also induce fission. Including a room, the neutron count rate has increased to 5.65 counts/second, closer to the measured 5.9 counts/second.

A standard measure of the amount of correlation in the count distribution from a fission source is the deviation from a Poisson distribution. The Feynman variance to mean is the simplest measure used in assay. The increased variance over a Poisson distribution is due to the large fluctuations in the number of neutrons emitted by fission chains. The normalization by the average number of counts determines a quantity that, for spontaneous fission initiated chains only, depends only on multiplication and efficiency. In this HEU problem, there is additional correlated contribution to the variance from large fluctuations in the number of neutrons created by cosmic ray induced burst processes (enhanced in SNM by fission chains), and in the mean by additional room background. (As we mentioned, the room background can also make a correlated contribution by initiating fission chains.)

We next show some comparisons between the simulations and data for the time gate dependent Feynman variance to mean. For simulations with spontaneous fission only, the comparison is shown below. 


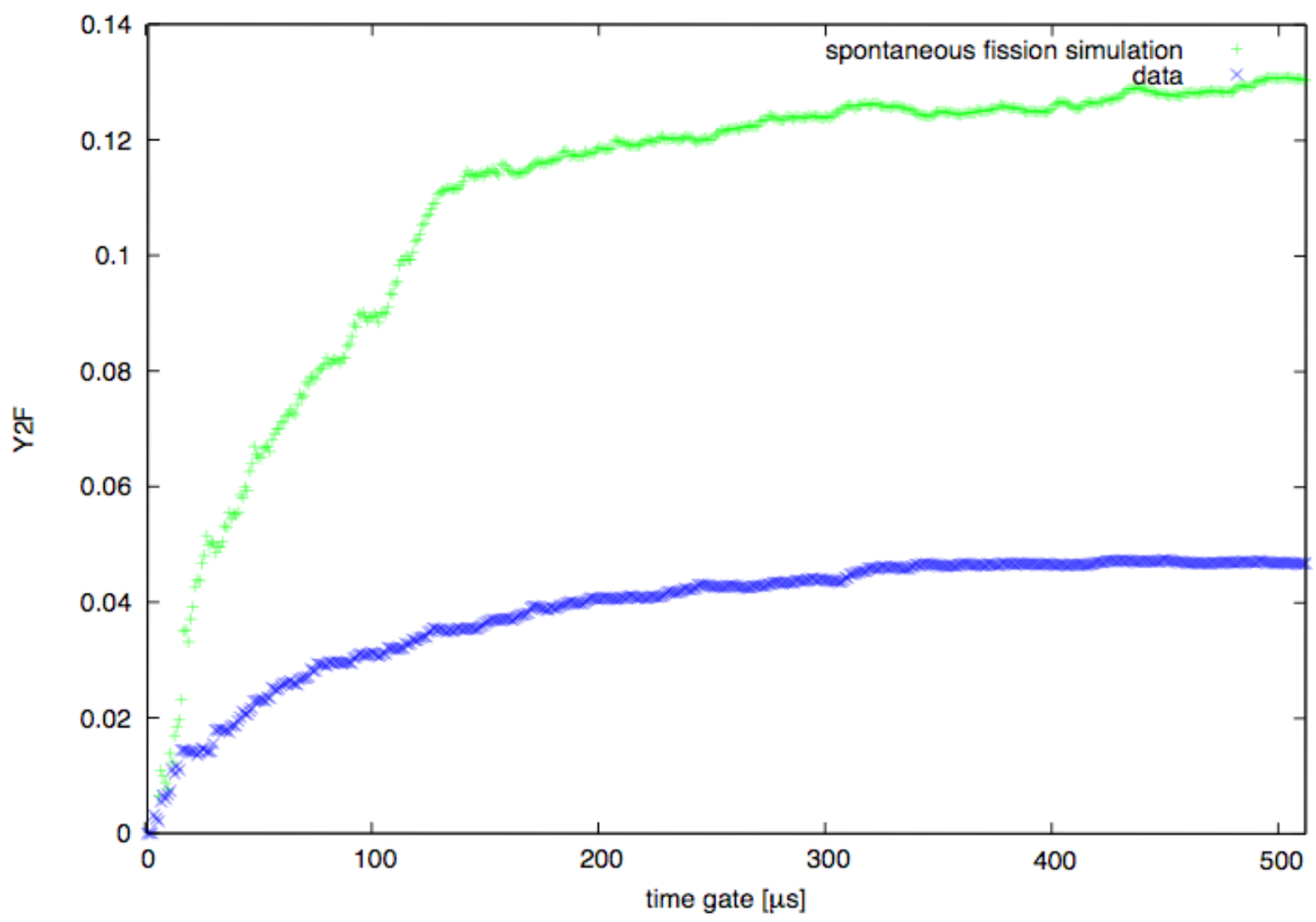

Figure 4: Time gate dependent Feynman variance to mean, for passive neutron counting of $\mathrm{HEU}$, from simulated spontaneous fission initiated fission chains (green) and from experimental data (blue). This quantity would be zero for all time gates (up to statistical fluctuations) for a random neutron source. The last data points for the $512 \mu$ s time gate are determined from the count distribution data of Figure 1. The time dependent approach to the long time gate asymptotic value is characteristic of the neutron diffusion time scale.

The simulated data is above the experimental data, not because of increased correlation, but because the count rate is almost 3 times higher in the experiment due to background. The higher count rate pulls down the magnitude of the increased variance that comes from correlated bursts.

When the cosmic rays and room are included the comparison is shown below. 


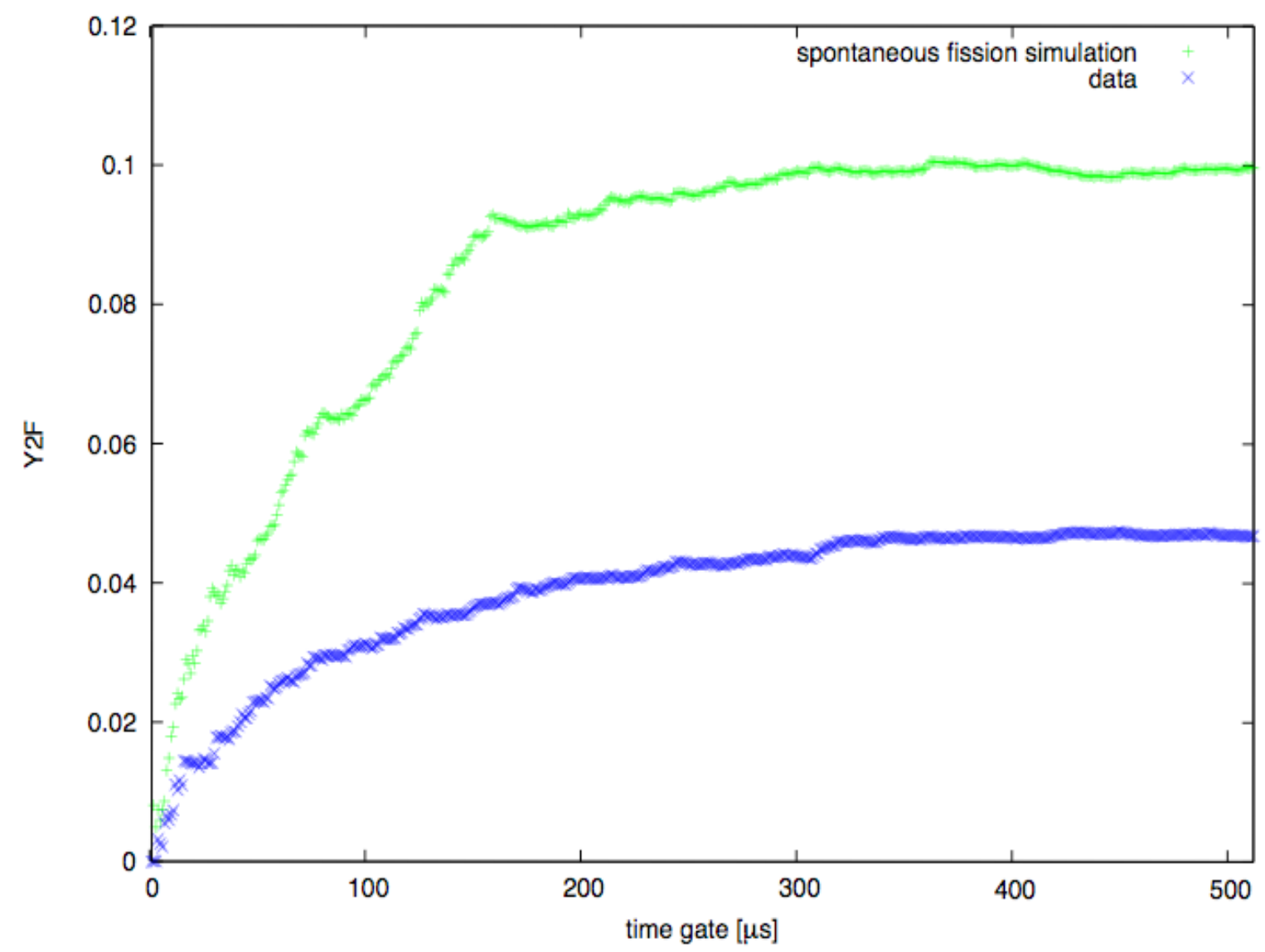

Figure 5: Time gate dependent Feynman variance to mean, for passive neutron counting of HEU, from simulation (green) and from experimental data (blue). This quantity would be zero for all time gates (up to statistical fluctuations) for a random neutron source. The last data points for the $512 \mu \mathrm{s}$ time gate are determined from the count distribution data of Figure 3 . The simulation includes spontaneous fission initiated chains, cosmic ray initiated bursts in $\mathrm{HEU}$, and cosmic ray created room background.

The simulation Feynman variance to mean is still larger than the experimental data, but slightly closer to the data as compared to Figure 4. The simulation including cosmic rays without the concrete room has a much larger discrepancy, with asymptotic value over 1.8. This is because of the significantly increased correlation, seen in the high multiplicity cosmic ray initiated events of Figure 2, combined with a count rate that is too low. With the room, the simulated count rate is closer to the experimental value, and the cosmic ray correlation is slightly suppressed, but still shows more correlation than the data. This comparison shows that for passive neutron counting of HEU of low multiplication, with intrinsically low statistics because of the low rate of fission chain initiation, cosmic ray events can significantly affect the interpretation of the signal. 


\section{Pu}

A $4.5 \mathrm{~kg}$ Pu ball moderated by 3" thick polyethylene was measured at 7300' altitude. The spontaneous fission rate of weapons grade $\mathrm{Pu}$ is about 30,000 spontaneous fissions/second per $\mathrm{kg}$ of $\mathrm{Pu}$. This moderated $\mathrm{Pu}$ ball has a multiplication of about 14 . The $512 \mu$ s time gate count distributions are now dominated by contributions from overlapping fission chains. An example of a simulation of the spontaneous fission initiated signal compared to the experimental count distribution data is shown below ${ }^{1}$.



Figure 6: Neutron count distribution data (blue) from moderated Pu ball, $512 \mu$ s time gate, compared to a simulation (green) that does not include the cosmic ray contribution. The red points are a Poisson distribution of the same count rate as the experimental data. The much broader distributions of both data and simulation are indicative of the very large fluctuations from highmultiplication fission chains.

Below we show a simulation of the cosmic ray contribution only, for 51.2 seconds of count time, the same count time as the experimental data. 




Figure 7: Simulation of $512 \mu$ s time gate neutron count distribution (green) from cosmic rays incident on the 3" polyethylene moderated Pu ball, compared to a the experimental count distribution. The cosmic ray induced count rate is $22.9 /$ second. Even at 7300 ' altitude of LANL, the cosmic ray induced burst contribution (seen only at low multiplicity) to the small Pu ball is meager in 51.2 seconds.

This simulation of the cosmic ray contribution to the neutron count distribution is dwarfed by the data. This simulation shows that even with the high flux of cosmic rays at 7,300' altitude of LANL, cosmic rays make only a perturbatively small contribution to the Pu spontaneous fission initiated fission chain contribution.

The combined spontaneous fission and cosmic ray induced contributions to the count distribution are shown below compared to the experimental data. 


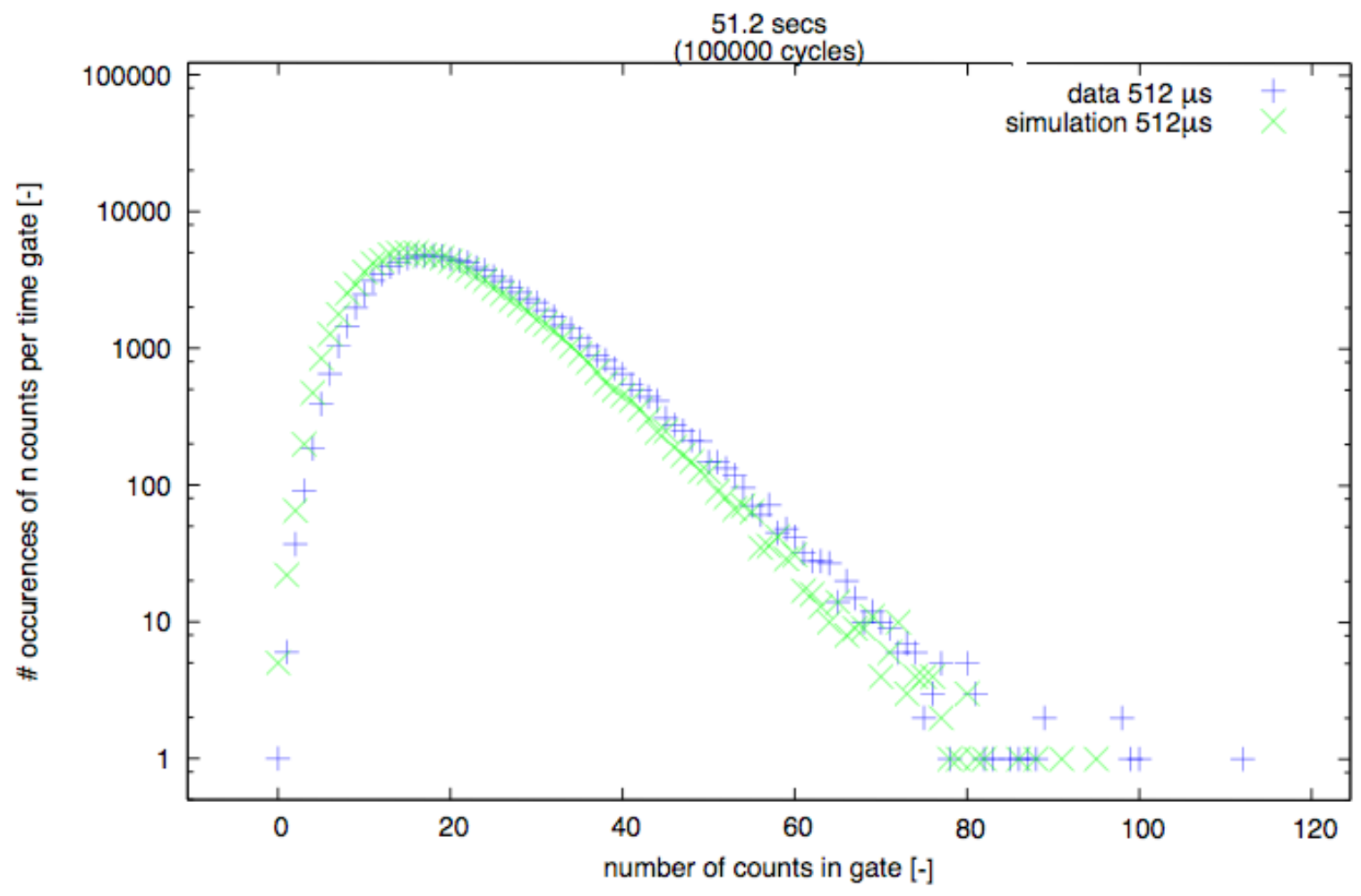

Figure 8: Neutron count distribution for Pu ball moderated by 3” thick polyethylene: simulation (green), including spontaneous fission induced chains and cosmic ray induced contribution, compared to experimental data (blue).

The remaining discrepancies between simulation and experimental data for this Pu object can be due to two contributions not included in the simulations, the presence of concrete walls in the room, and additional sources of neutrons from $(\alpha, n)$ reactions; $\gamma$-rays from the ${ }^{9} \mathrm{Be}(\alpha, n){ }^{12} \mathrm{C}$ reaction are observed. Both these contributions will move the simulation result closer to the data.

\section{Conclusions and Next Steps}

For HEU the passive fission chain neutron signal has a significant cosmic ray contribution. The passive fission chain signal from HEU is so weak that the count rate is dominated by cosmic rays interacting with the room. The high-energy cosmic ray hadrons contribute to initiating fission chains, and dominate the largest burst contribution. For Pu the cosmic ray contribution is very small. 
Fission chains are characterized by enormous fluctuations in the number of created neutrons from one chain to the next. For HEU the rate of initiating chains is very low; in this example there are only about 85,000 spontaneous fissions in over 1.7 hours of counting, restricting the data to events with probability not much less than $10^{-5}$. For the low multiplication of this shell of about 2.1 (the average number of neutrons created starting a single neutron, although on average 0.4 of these are internally absorbed to induce fissions in the chain), the probability to get 6 counts from a spontaneous fission initiated fission chain with $4 \%$ detection efficiency is about $10^{-5}$. ( $\sim 50$ neutrons had to have been created by a chain from which 6 are counted.) The cosmic ray events are also statistical, dependent on exotic astrophysical processes. Consequently the HEU data can have large fluctuations.

To better analyze the statistical fluctuations in these comparisons, it would be useful to perform new measurements of the HEU shell and more completely model the experiments. For the $\mathrm{Pu}$, it is known that there is beryllium present, probably only on the surface; the $\operatorname{Be}(\alpha, n)$ contribution should be included in the modeling.

To apply this method to the transparency context, we would like to do measurements and simulations of SNM in storage containers, and especially in high neutron background environments that may reflect real storage facility conditions. It is likely that there is some possible background count rate where the fission initiation rate from background would begin to dominate the signal completely (as in the case of multiplying $\mathrm{Pu}$ ). It would be desirable to study where this comes to pass in order to understand this signal as fully as possible. Fortunately this condition can be simulated in measurement too by placing external sources near the multiplying objects. All of these measurements and comparisons to simulations should be done in order to fully understand this very interesting problem. It is also possible that by controlling where the measurements are made (near or away from background or a combination of both) a maximum amount of information can be derived. We could also measure and simulate HEU combined with depleted uranium, perhaps composite HEU and Pu parts, and with shielding. These measurements should be studied with both thermal neutron $\left({ }^{3} \mathrm{He}\right)$ and fast neutron counters.

Thermal neutron count distribution methods with ${ }^{3} \mathrm{He}$ described here are certainly adequate for plutonium, and we believe, despite the large cosmic ray contribution for highly multiplying HEU. For low multiplying HEU, or 
for Pu oxide with light element impurities, we believe methods based on fast neutron and $\gamma$-ray counting with nanosecond time resolution are potentially far superior. We have demonstrated nanosecond counting using liquid scintillators for which the neutron and $\gamma$-ray contributions can be separated using pulse shape discrimination, with high fidelity. Each neutron creation event can be isolated. From the correlation of neutrons and $\gamma$-rays we believe it is possible to at least statistically distinguish fission from $(\alpha, \mathrm{n})$ reactions.

\footnotetext{
${ }^{1}$ J.M. Verbeke, A. Dougan, L.F. Nakae, K.E. Sale, and N.J. Snyderman, "Neutron Correlations in Special Nuclear Materials, Experiments and Simulations,” UCRL-PROC-231582.

2 J.M. Verbeke, C. Hagmann, D. Wright, "Simulation of Neutron and Gamma Ray Emission from Fission,” UCRL-AR-228518-REV-1; http://nuclear.llnl.gov/simulation ; "Cosmic-Ray Shower Generator (CRY) for Monte Carlo Transport Codes,” C. Hagmann, D. Lange, and D. Wright, 2007 IEEE Nuclear Science Symposium Conference Record, Oct27-Nov3, Honolulu, Hawaii.

${ }^{2}$ G. Chapline, C. Hagmann, P. Kerr, N. Snyderman, and R. Wurtz, "Cosmic Ray Induced Neutron Bursts in a Lead Pile,” UCRL-TR-230895.
} 